\title{
ATOMIC FORCE MICROSCOPY \\ OF INSTABILITIES AND REORGANIZATION OF LANGMUIR-BLODGETT FILMS
}

\author{
D.Y. Takamoto, E. Ter-Ovanesyan, D.K. Schwartz, R. Viswanathan, \\ S. Chiruvolu and J.A. ZaSadzinsKi* \\ Departments of Chemical Engineering and Materials \\ University of California, Santa Barbara, CA 93106, USA
}

\begin{abstract}
The atomic force microscope was used to show that Langmuir-Blodgett films are unstable to reorganization via a folding mechanism by which uniformly thick films spontaneously form holes and multilayer steps. These bilayer step defects originate at isolated sites and quickly spread to cover the entire film. The defects retain the sixfold symmetry of the underlying molecular lattice; after sufficient time, straight edges begin to form and the entire film is comprised of high islands with straight edges aligned with sixfold symmetry. The kinetics of the reorganization depend strongly on the chain length of the fatty acid used and the nature of the substrate. The results suggest that the reorganization is driven by an interconversion of strained, asymmetric monolayers to unstrained, centrosymmetric bilayers.
\end{abstract}

PACS numbers: 82.65.Dp

\section{Introduction}

Langmuir-Blodgett (LB) films, layered assemblies of amphiphilic molecules, have potential applications in the areas of non-linear optics, molecular electronics, biosensors, and as models for cell membranes [1]. Most of these applications rely upon the self-organization of amphiphilic molecules to form very thin and essentially perfect films with known thickness and molecular orientation. Although a few experimenters have warned that the flat, featureless LB film may not be the equilibrium structure [2], little work has been done to study the approach to equilibrium as the tools for this study had not been available until the development of scanning probe microscopy. Any metastability is detrimental, for example, to applications of LB films in non-linear optics where interdiffusion and molecular rearrangement can severely diminish second harmonic generation, which depends explicitly on molecular orientation and layering [3, 4]. Probe microscopy

*To whom correspondence should be addressed. 
techniques have clear and significant advantages over scattering and spectroscopy when it comes to exploring such inhomogeneities and defects [5-7]. Conventional contact-mode atomic force microscope (AFM) has emerged as the preferred technique for imaging LB films [5-7] because it can probe LB films quickly, directly, and non-destructively without the need for high vacuum or metal replicas as is the case for electron or scanning tunneling microscopy [8]. Furthermore, we can vary the magnification from molecular resolution images to a field of view of $100 \mu \mathrm{m}$, allowing the correlation of structures on extremely different length scales.

We have chosen cadmium fatty acid salts as the materials for this study as they have been ubiquitous to LB deposition schemes since the days of Langmuir and Blodgett, and are still commonly used as spacer layers for non-linear optics [3, 4]. The conclusion of the present work is that a homogeneous and uniformly thick Langmuir-Blodgett film, when allowed to equilibrate under water, will reorganize to form steps and holes with heights corresponding to integral numbers of bilayers. We believe that the driving force behind this reorganization is the elimination of non-centrosymmetric layers - a combination of AFM, electron and X-ray diffraction $[5,9]$ shows that monolayers are inherently less ordered than bilayers. The continued approach to equilibrium involves the formation of macroscopic crystallites whose straight edges are explicitly shown to correspond to molecular lattice symmetry directions. The crystallites appear to retain the hexagonal symmetry of the original LB films, indicating that the reorganization is a bulk folding of layers rather than molecular scale events. The minimum size of the folded domains likely depends on the competition between the increase in cohesion energy of the newly folded regions and the extra energy associated with the increase in hydrocarbon-water contact at newly formed step edges. These ideas are reinforced by the fact that the kinetics of the reorganization depend strongly on the chain length of the fatty acid salt and the nature of the substrate in contact with the monolayer.

\section{Materials and methods}

Palmitic, stearic, arachidic and behenic acids $\left(\mathrm{CH}_{3}\left(\mathrm{CH}_{2}\right)_{14-20} \mathrm{COOH}\right.$, Aldrich, 99\%) were spread quantitatively from chloroform (Fisher spectranalyzed) solution onto an aqueous [water from a Milli-Q (Millipore Corporation, Bedford, MA) system was used] subphase in a commercial NIMA (NIMA Technology, Ltd., Warwick Science Park, Coventry CV4 7EZ, England) trough. The subphase water included $5 \times 10^{-4} \mathrm{M} \mathrm{CdCl}_{2}$ (Aldrich $99.99 \%$ ) and was adjusted to a $\mathrm{pH}$ of 6.5 by addition of $\mathrm{NaHCO}_{3}$ (Aldrich $99.95 \%$ ). Substrates were freshly cleaved mica or polished silicon wafers (Semiconductor Processing, Boston, MA) (orientation (100), $3 \mathrm{ohm} \mathrm{cm}, n$-type) with a rms roughness of approximately $3 \AA$ as measured by AFM. Prior to deposition, the silicon wafers were cleaned in a hot solution of $\mathrm{H}_{2} \mathrm{O}_{2} / \mathrm{H}_{2} \mathrm{SO}_{4}$ (3:7 ratio) to remove any organic contaminants while leaving the amorphous native oxide intact, then stored in clean water until use. The mica substrates were cleaned by continuous rinsing with ethanol for 5 min. After removal from the ethanol bath, the mica was cleaved using ordinary adhesive tape, and inserted into the subphase. Since all films were $\mathrm{Y}$ type (i.e. adjacent layers stack head to head or tail to tail), films deposited on a hydrophilic substrate and 
imaged in air had an odd number of layers $(1,3$, or 5$)$ with the methyl end of the alkyl chain at the interface. Isotherms and film deposition were done on the NIMA trough at $22.0 \pm 0.5^{\circ} \mathrm{C}$ and a surface pressure of $\pi=30 \pm 0.1 \mathrm{dyn} / \mathrm{cm}$. Film transfer was accomplished by vertical dipping at a speed of approximately $1.6 \mathrm{~mm} / \mathrm{min}$. Transfer ratios were approximately unity. Aging under aqueous subphase was achieved by interrupting the dipping process with the film submerged, waiting for a given time, and then drawing the substrate through the air-water interface. This last pass through the interface could be done with or without a monolayer at the interface. Films were stored in closed containers for times ranging between 1 and 30 days before imaging. The length of time between deposition and imaging did not affect the images.

AFM measurements were performed with a Nanoscope Multimode (Digital Instruments, Inc., Goleta, CA 93117) FM in air at room temperature, using a $15 \mu \mathrm{m}$ or a $1 \mu \mathrm{m}$ scan head and a silicon nitride tip on a cantilever with a spring constant of $0.12 \mathrm{~N} / \mathrm{m}$. Most imaging was done using "height" mode (utilizing feedback to keep the spring at constant deflection and measuring sample motion), however, the best molecular resolution was achieved by using "force" mode, i.e. scanning the tip at approximately constant height and measuring spring deflection. Typical forces used were on the order of $10 \mathrm{nN}$. Further details about molecular resolution measurements have been given elsewhere $[5,6]$.

\section{Results}

Figure 1 shows an overview of the time evolution of a cadmium arachidate LB film when allowed to stay submerged under aqueous subphase. Figure 1a shows a film submerged for about $10 \mathrm{~min}$. Small volcano-like patches were observed which appeared to be nucleation sites for reorganization. Three heights are observed, corresponding to 1,3 , and 5 layer regions. The nucleation site appears as though small bilayer sections have simply peeled off the substrate and flipped over onto already covered areas of the film to form multilayer patches. The sizes and shapes of the holes in the film are consistent with the sizes and shapes of the multilayer regions.

After 30 min. (Fig. 1b), the process is so advanced that nearly the entire film is involved in the reorganization. There is an extensive network of multilayered terraces, separated by heights that are a multiple of the bilayer spacing of $5.6 \mathrm{~nm}$ [5]. From a rough analysis of a number of images, it is apparent that the relative areas with less than the average number of layers is roughly equal to those with more than the average number of layers. Hence, none of the cadmium arachidate is being lost or solubilized, it is merely reorganizing on the surface. After 10 hours submerged (Fig. 1c), the pattern is much more dendritic in form and the shape of the steps is much more anisotropic. After 48 hours submerged (Fig. 1d) the film has segregated into isolated islands, separated by regions of monolayer coverage. Not only do the islands have straight, sharp boundaries, but, as shown by the approximately hexagonal symmetry of the Fourier transform (inset), the island edges have well defined absolute orientations.

Because of the large range of magnifications available to the AFM we have previously [6] been able to explicitly establish a correspondence between the di- 

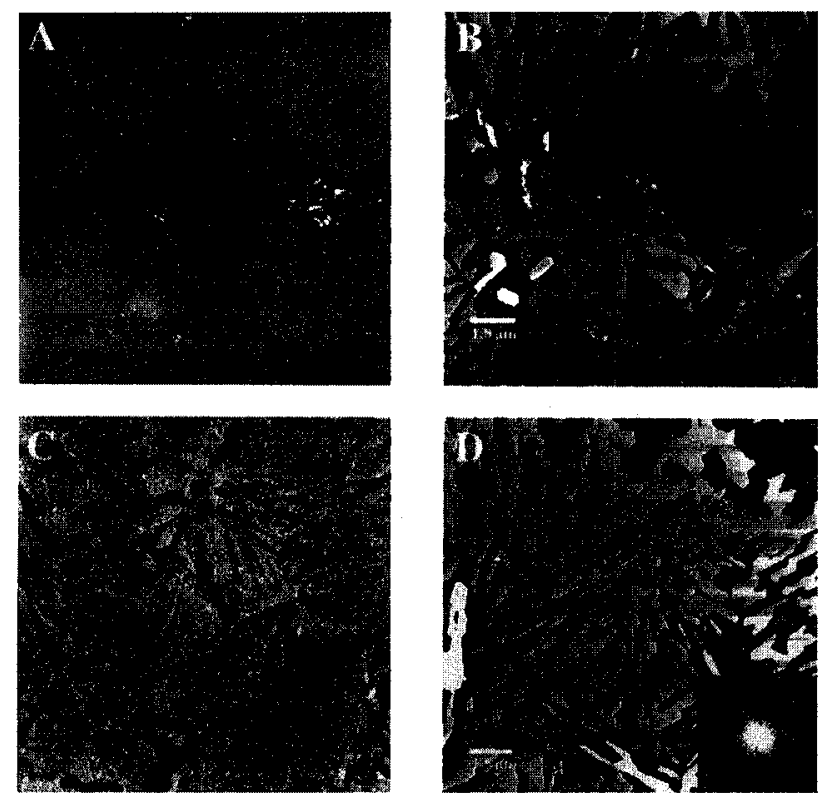

Fig. 1. AFM images $(15 \mu \mathrm{m} \times 15 \mu \mathrm{m})$ of LB films of cadmium arachidate after being aged for various periods of time under aqueous subphase. (a) A 3-layer film on silicon after $10 \mathrm{~min}$ of aging between layers 2 and 3. Several nucleation sites for the reorganization are visible. (b) A 3-layer film on silicon aged for $30 \mathrm{~min}$ between layers 2 and 3. The reorganization is extensive and appears to happen by folding over parts of the monolayer to form multilayers. (c) A 5-layer film on silicon aged $10 \mathrm{~h}$ between layers 4 and 5 . Long, straight features begin to appear. (d) A 5-layer film on silicon aged $48 \mathrm{~h}$ between layers 4 and 5 . The film consists completely of distinct islands with straight edges. The inset Fourier transform shows that the island edges have an approximate sixfold symmetry.

rection defined by the edge of an island and the molecular lattice direction within that island. For all the islands we observe (Fig. 1d), the [01] direction is identical to the edge direction observed. Due to the nearly hexagonal symmetry of the lattice $[5,6]$, most of the crystallites in the film are related by a rotation of about $60^{\circ}$. The large anisotropy of the crystallites formed during the reorganization process suggests that the [01] lattice direction has a substantially lower edge energy than do the other crystallographic directions. In the rectangular packing of cadmium arachidate, the [01] direction has the shorter of the two lattice vectors $[5,6]$ and hence is the close packed direction of the lattice.

The kinetics of the reorganization is strongly affected by the chain length of the fatty acid in the films. Figure $2 \mathrm{a}$ shows a five-layer film of cadmium palmitate (16 carbon fatty acid) after just $10 \mathrm{~min}$ in the subphase. The extent of reorganization is comparable to that of cadmium arachidate after 48 hours (Fig. 1d). Figure $2 \mathrm{~b}$ shows a cadmium arachidate five-layer film on silicon that shows less extensive reorganization than the palmitate after the same ten minutes in the subphase. However, cadmium behenate (22 carbon fatty acid) shows only isolated 

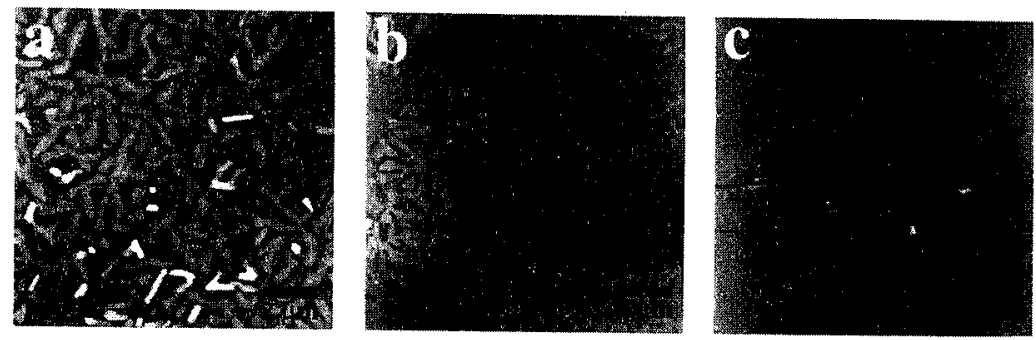

Fig. 2. $12 \times 12 \mu \mathrm{m}^{2}$ images of (a) cadmium palmitate (16 carbons) (b) cadmium arachidate (20 carbons) and (c) cadmium behenate (22 carbons) nominally five-layer films on silicon after have been aged for $(a, b)$ ten minutes or (c) one hour in the subphase to show the effects of fatty acid chain length. In (a) the previously uniform films have been thoroughly disrupted and have reorganized into small multilayer islands. The extent of reorganization is comparable to that of a cadmium arachidate film after $48 \mathrm{~h}$ (Fig. 1d). The cadmium arachidate film in (b) shows extensive folding and the entire film is involved in the reorganization. In (c) the reorganization is much slower - all that happens is the formation of isolated eruption sites that indicate the first stages of the layer folding of the cadmium behenate. However, given sufficient time, all the films reorganize to form patterns similar to Fig. 1d.
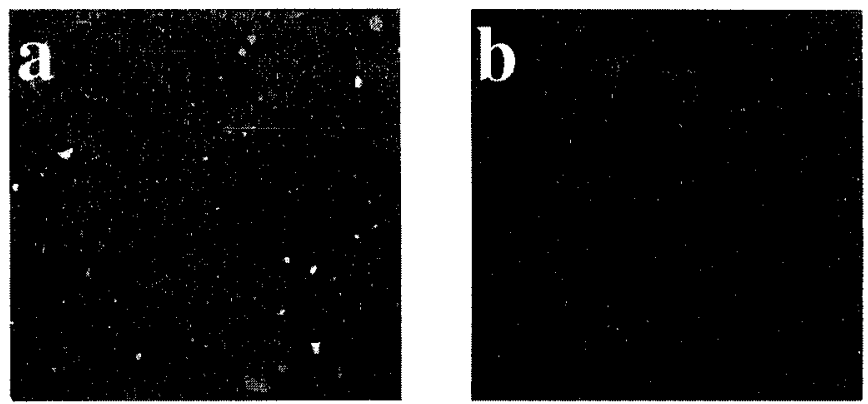

Fig. 3. $15 \times 15 \mu \mathrm{m}^{2}$ AFM images of (a) cadmium arachidate three-layer films on oxidized silicon and (b) on mica substrates. After $30 \mathrm{~min}$ in the subphase, the film on oxidized silicon (a) is thoroughly reorganized, while the same three-layer film on mica (b) shows only small pinhole defects and is effectively flat. The cadmium ions preferentially interact with the anionic mica surface rather than the neutral oxidized silicon; however, these images show that the monolayer is important to the reorganization process.

nucleation sites after even one hour in the subphase (Fig. 2c). Multilayer films of all three fatty acids films have the same crystal lattice when examined in air [5], so it is likely that the formation of island edges at which the hydrocarbon portion of the fatty acid are exposed to the subphase limits the kinetics of formation of the reorganized structures. 
The reorganization also depends on the nature of the substrate [10-12]. Figure $3 \mathrm{a}$ shows an originally five-layer cadmium arachidate film on an oxidized silicon wafer after $30 \mathrm{~min}$ in the subphase. The reorganization is well advanced. However, Fig. $3 \mathrm{~b}$ shows that a similar five-layer film on a mica substrate is essentially flat except for a small density of pinholes. This is surprising in that the choice of substrate did not influence the lattice parameters and symmetry of multilayer films of fatty acid salts [5]. However, it does suggest that there is something about the monolayer and its attachment to the substrate that is influencing the stability of the film structure.

In all of our studies to date, we have never observed any lattice structure in fatty acid salt monolayer films on any substrate, suggesting that the monolayer is inherently different in structure than multilayer films. To extend our AFM observations, we performed electron diffraction on 1- and 3-layer films on a hydrophilic formvar substrate. The diffraction pattern from the 3-layer film (Fig. 4a) agrees quantitatively with our AFM results. The diffraction pattern from the 3-layer films shows sharp reflections, higher order reflections and evidence of twinning, indicative of long-range orthorhombic packing, as do AFM images [5, 6]. This is evident in the large difference between the width of the spots along the radial direction (which is indicative of molecular ordering) and the azimuthal direction (which is indicative of the orientation of the crystalline grains in the films). The diffraction from the monolayer, however (Fig. 4b), agrees with other electron and X-ray diffraction data [9] and shows a hexagonal structure with a nearest-neighbor dis-
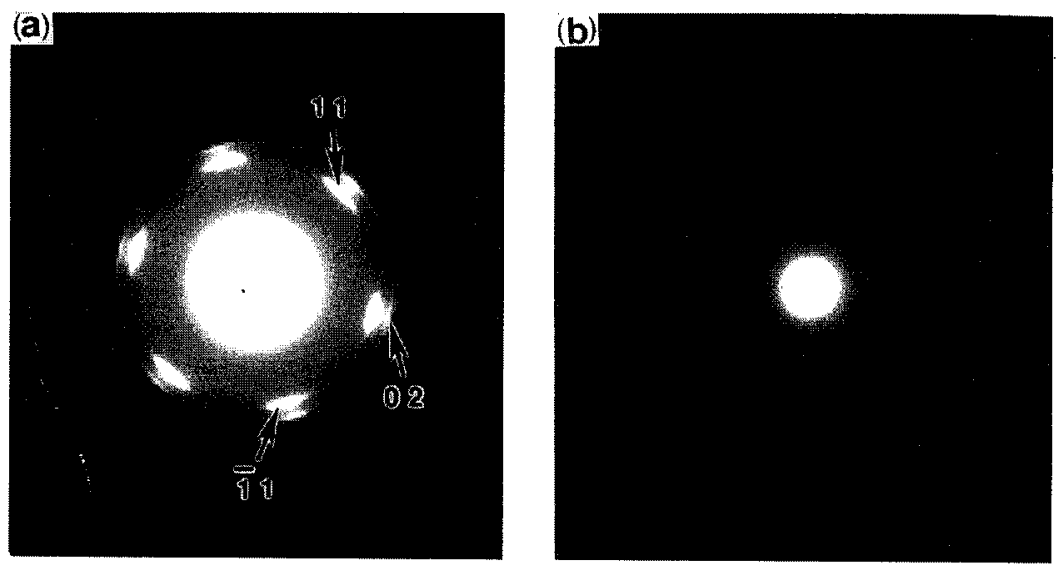

Fig. 4. Electron diffraction patterns of (a) five-layer and (b) monolayer films of cadmium arachidate on hydrophilic formvar substrates. The multilayer films show the orthorhombic structure (the main reflections are highlighted) and the twinning typically observed in multilayer films. Higher order reflections, indicative of long-range order are readily apparent. In the monolayer, weak and broad hexagonal reflections are observed, consistent with short-range order. The area per molecule in the multilayer films is $18 \AA^{2}$, while in the monolayer it is $19.4 \AA^{2}$. This suggests that the packing in the monolayer is very strained in comparison to the multilayer. 
tance of $0.47 \mathrm{~nm}$, giving an area per molecule of $19.4 \AA^{2}$, about $8 \%$ larger than in multilayer films. The monolayer reflections are almost as wide in the radial direction as in the azimuthal direction, indicating much less molecular order in the monolayer. The extra area per molecule of the monolayer film and the short-range positional correlation length [9] implies that there is considerable motion and disorder within the individual alkane chains as compared to the close packed chains in the multilayer (area per molecule of $18 \AA^{2}$ ). This is supported by Fourier transform infrared (FTIR) spectroscopy measurements [9] which show that the alkane chains of monolayers show less crystallinity than in thicker films. The electron diffraction pattern shows the average position of the centers of mass of the chains, which have hexagonal symmetry but no long-range ordering. This increased disorder in the alkane chains of monolayers is consistent with our AFM images that show an absence of order at the methyl end of the chains. The end of the fatty acid chains observed by the AFM are the least ordered part of the chain and increased motion and disorder leads to a complete lack of order in the AFM images.

\section{Discussion}

In previous work $[5,6]$ we have noted that multilayer films of fatty acids salts have well defined and long-ranged orthorhombic lattices, while monolayer films of the same salts are disordered. The variation between the monolayer and multilayer structure may be the driving force for the instabilities seen here. We can rule out purely entropic effects (which are important in thermal crystal roughening [10]) because the state the system appears to be approaching in equilibrium is one of thick, but flat, crystallites. If the reorganization were driven by entropic considerations we would expect the film to approach a much rougher final state.

By stacking higher, the film can increase the number of internal bilayers while minimizing both substrate and surface monolayers. In bulk fatty acid crystals, the equilibrium structure consists of a monolayer of metal ions sandwiched between bilayers of fatty acids [13], or a centrosymmetric structure. However, the LB technique deposits films that are inherently asymmetric - at the air-water interface on the Langmuir trough, the cations are associated with the aqueous phase and the hydrocarbon chains of the fatty acid are oriented toward the air. The main difference between the internal layers of the film and the layer in contact with the substrate (or the layer in contact with the water) is the inability of the first layer to form a centrosymmetric structure. From the electron diffraction and AFM images, the asymmetric monolayers are less ordered than the centrosymmetric bilayers; the area per molecule is about $8 \%$ larger in the monolayer, and the symmetry is hexagonal rather than orthorhombic; the absence of higher-order reflections and the broadening of the reflections observed show that the order is short-ranged and highly defective. This suggests substantial packing frustration in the monolayer that is relieved in the centrosymmetric bilayer [12]; the asymmetric structure likely is highly strained as the area occupied by the chains $\left(19.4 \AA^{2}\right)$ is larger than the equilibrium area in the bulk crystals and multilayers $\left(18 \AA^{2}\right)$. By folding over, the strained, asymmetric monolayers can relax to the equilibrium, unstrained and ordered centrosymmetric bilayer, thereby relieving the frustration. 
The strong dependence of the reorganization kinetics on the nature of the substrate show that the stability of the monolayer is important to the reorganization process. The cadmium cation can interact strongly and specifically with the negatively charged mica surface; the cadmium ions do not interact with the neutral silicon oxide surface. Hence, monolayers on mica are expected to be more stable than monolayers on silicon oxide. The stronger the interaction between the substrate and the monolayer, the more stable the films should be. Future work will concentrate on lead films that show a strained van der Waals epitaxial growth on mica [14]; these films should reorganize much more slowly than the cadmium arachidate films.

An additional energetic cost are the island edges that have a repulsive hydrophobic interaction between the exposed hydrocarbon chains of the cadmium arachidate and the subphase water. However, it is possible that the island edges are closed off in a sort of semi-micellar or other arrangement to minimize this cost. The cost of edges is also not uniform; the large shape anisotropy of the crystallites is consistent with the fact that the [01] direction is preferred to all others. Changing the length of the hydrocarbon chain, and hence the strength of the hydrophobic interaction and resulting edge energy, alters the kinetics of the reorganization dramatically. Cadmium palmitate (16 carbons) reached a degree of reorganization comparable to a 48 hour aged cadmium arachidate (20 carbons) film after only 10 min aging. Cadmium behenate (22 carbons) needed approximately $60 \mathrm{~min}$ aging to reach the stage of first nucleation that was seen in cadmium arachidate after 10 min aging. Decreasing the edge energy by decreasing the length of the fatty acid hydrocarbon chain clearly decreases the minimum size of a nucleation site, resulting in the faster kinetics. However, given sufficient time, all of the films reorganized regardless of chain length.

This edge energy serves to explain, however, why both nucleation and crystal growth takes place by the movement and folding of areas large in comparison to molecular dimensions. The ratio of surface area to edges is roughly proportional to the square root of the surface area. Hence, the edge energy for a small island would be disproportionately large. As a consequence, there is likely to be a minimum size island required to initiate folding and nucleate the reorganization, similar to the case in three dimensions for condensation of a liquid from its vapor in which a minimum size droplet is necessary to initiate nucleation of a liquid in a vapor due to the competition between the latent heat of condensation and surface tension [12].

\section{Conclusion}

We have observed the evolution of LB thin films of cadmium fatty acid salts when allowed to age under aqueous subphase. The uniform film reorganizes by forming terraces of bilayer steps initially in a random network. After more time the film forms into separate island crystallites (with straight edges) that have orientations related by an approximate hexagonal symmetry. The predominant direction of the island edges corresponds to the [01] molecular lattice direction. The crystallites appear to retain the hexagonal symmetry of the original LB films, indicating that the reorganization is a bulk folding of layers rather than molecular scale events. The driving force for the reorganization can be attributed to the 
elimination of strained, asymmetric monolayers in favor of unstrained, centrosymmetric bilayers [13]. This effect is of great practical significance to those researchers interested in building up multilayers of different chemistry or functionality using Langmuir-Blodgett deposition. The asymmetric layers of fatty acids deposited by the LB technique are inherently metastable. The layer ordering and orientation imposed by the deposition are severely disrupted as the film coated substrate is allowed to traverse the subphase, for example, during the time required to aspirate one type of monolayer from the surface and replace it with another or even just the normal time required for multilayer deposition. The reorganization we see is one way for the system to eliminate strained and asymmetric monolayers in favor of unstrained and centrosymmetric bilayers. The LB technique is incompatible with depositing a centrosymmetric structure - reorganization is an inevitable consequence of the progression to equilibrium. As a practical matter, if such alternate layered systems are required, cadmium behenate, with its substantially slower reorganization kinetics, should be chosen over cadmium arachidate or cadmium stearate. However, the main conclusion is that the asymmetric monolayers deposited by the Langmuir-Blodgett process are inherently unstable to centrosymmetric bilayers, which limits the applications of the LB process. Alternate molecular geometries and deposition strategies will be required to provide satisfactory layered materials.

\section{Acknowledgments}

This work was supported by the National Institutes of Health under grant \#GM 47334, the NSF under grant CTS-9634050. We also thank Frank Grunfeld of NIMA Technologies for his assistance with the trough and software.

\section{References}

[1] G.G. Roberts, Adv. Phys. 34, 475 (1985).

[2] G.L. Gaines, Thin Solid Films 68, 1 (1980); F. Kopp, U.P. Fringeli, K. Mühlethaler, H. Günthard, Biophys. Struct. Mechanism 1, 75 (1975).

[3] L.M. Hayden, S.T. Kowel, M.P. Srinivasan, Opt. Commun. 61, 351 (1987).

[4] M. Shimomura, K. Song, J.F. Rabolt, Langmuir 8, 887 (1992).

[5] D.K. Schwartz, J. Garnaes, R. Viswanathan, J.A. Zasadzinski, Science 257, 508 (1992); D.K. Schwartz, J. Garnaes, R. Viswanathan, S. Chiruvolu, J.A. Zasadzinski, Phys. Rev. E 47, 452 (1993); J.A. Zasadzinski, R. Viswanathan, L. Madsen, J. Garnaes, D.K. Schwartz, Science 263, 1726 (1994); J. Garnaes, D.K. Schwartz, R. Viswanathan, J.A. Zasadzinski, Nature 357, 54 (1992).

[6] D.K. Schwartz, R. Viswanathan, J.A. Zasadzinski, J. Phys. Chem. 96, 10444 (1992).

[7] E. Meyer, L. Howald, R.M. Overney, H. Heinzelmann, J. Frommer, H.-J. Güntherodt, T. Wagner, H. Schier, S. Roth, Nature 349, 398 (1991); J.A.N. Zasadzinski, C.A. Helm, M.L. Longo, A.L. Weisenhorn, S.A.C. Gould, P.K. Hansma, Biophys. J. 59, 755 (1991); L. Bourdieu, P. Silberzan, D. Chatenay, Phys. Rev. Lett. 67, 2029 (1991).

[8] J.A. Zasadzinski, J. Schneir, J. Gurley, V. Elings, K.P. Hansma, Science 239, 1013 (1988); J.T. Woodward, J.A. Zasadzinski, Phys. Rev. E 53, R3044 (1996); J.T. Woodward, J.A. Zasadzinski, Biophys. J.,72, 964 (1997). 
[9] S. Garoff, H.W. Deckman, J.H. Dunsmuir, M.S. Alvarez, J. Phys. (France) 47, 701 (1985); A. Bonnerot, P.A. Chollet, H. Frisby, J. Hoclet, Chem. Phys. 97, 365 (1985); C. Boehm, R. Seitz, H. Riegler, Thin Solid Films 178, 511 (1989); P. Tippmann-Krayer, R.M. Kenn, H. Moewald, Thin Solid Films 210, 577 (1992).

[10] M. Wortis, in: Chemistry and Physics of Solid Surfaces VII, Eds. R. Vanselow, R. Howe, Springer-Verlag, Berlin 1988, p. 367.

[11] H.G. Hansma, S.A.C. Gould, P.K. Hansma, H.E. Gaub, L.M. Longo, J.A. Zasadzinski, Langmuir 7, 1051 (1991); R. Viswanathan, D.K. Schwartz, J. Garnaes, J.A. Zasadzinski, Langmuir 8, 1603 (1992).

[12] J.N. Israelachvili, Intermolecular and Surface Forces, Vol. 2, Academic Press, London 1992.

[13] R.W. Corkery, S.T. Hyde, Langmuir 12, 5528 (1996); T.A. Barberka, U. Hohne, U. Peitsch, T.H. Metzger, Thin Solid Films 244, 1061 (1994); R.D. Vold, G.S. Hattiangid, Ind. Eng. Chem. 41, 2311 (1949); J.F. Stephens, C. Tuck-Lee, J. Appl. Crystallogr. 2, 1 (1969).

[14] R. Viswanathan, J.A. Zasadzinski, D.K. Schwartz, Science 261, 449 (1993). 